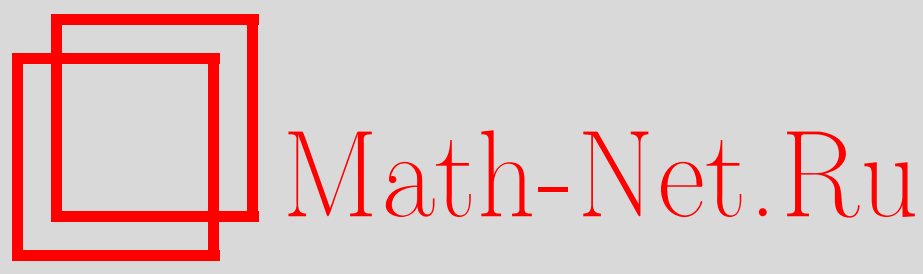

М. Гнатич, В. М. Хмара, В. Ю. Лазур, А. К. Рейтий, Метод ВКБ для квантово-механической задачи двух кулоновских центров, ТМФ, 2017, том 190, номер 3, 403-418

DOI: https://doi.org/10.4213/tmf9132

Использование Общероссийского математического портала Math-Net.Ru подразумевает, что вы прочитали и согласны с пользовательским соглашением http://www . mathnet.ru/rus/agreement

Параметры загрузки:

IP : 3.81 .55 .215

26 апреля 2023 г., 13:37:03

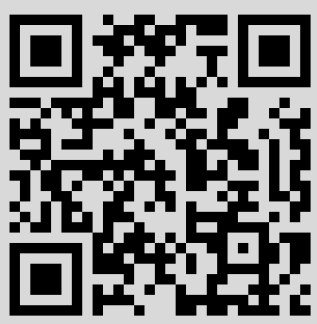




\title{
ФИЗИКА
}

Том 190, № 3

март, 2017

(C) 2017 r.

М. Гнатич*, В. М. Хмара ${ }^{\dagger}$, В. Ю. Лазур ${ }^{\ddagger}$, А. К. Рейтий ${ }^{\ddagger}$

\section{МЕТОД ВКБ ДЛЯ КВАНТОВО-МЕХАНИЧЕСКОЙ ЗАДАЧИ ДВУХ КУЛОНОВСКИХ ЦЕНТРОВ}

\begin{abstract}
$\mathrm{C}$ помощью модифицированной теории возмущений получены асимптотические выражения для двухцентровых квазирадиальной и квазиугловой волновых функций при больших межъядерных расстояниях $R$. Показано, что в каждом порядке по $1 / R$ поправки к волновым функциям выражаются через конечное число кулоновских функций с модифицированным зарядом. Для первой, второй и третьей поправок выведены простые аналитические выражения. Разработана последовательная схема получения разложений ВКБ для решений квазиуглового уравнения в квантово-механической задаче двух кулоновских центров. В рамках этой схемы построены квазиклассические двухцентровые волновые функции при больших расстояниях между фиксированными положительно заряженными частицами (ядрами) для всего пространства движения частицы с отрицательным зарядом (электрона). Метод обеспечивает простые равномерные оценки для собственных функций при произвольно больших межъядерных расстояниях $R$, включая $R \gg 1$. В отличие от теории возмущений квазиклассическое приближение не связано с малостью взаимодействия и поэтому имеет более широкую область применимости, что позволяет исследовать качественные закономерности в поведении и свойствах квантово-механических систем.
\end{abstract}

Ключевые слова: квазиклассическое приближение, метод ВКБ, два кулоновских центра, асимптотические решения.

DOI: https://doi.org/10.4213/tmf9132

Работа выполнена при финансовой поддержке Министерства образования и науки РФ (соглашение № 02.a03.21.0008), а также Ministry of Education, Science, Research and Sport of the Slovak Republic (грант VEGA 1/0345/17).

*Российский университет дружбы народов, Москва, Россия. E-mail: hnatic@saske.sk

${ }^{\dagger}$ Institute of Physics, Faculty of Sciences, Pavol Jozef Šafárik University in Košice, Košice, Slovakia. E-mail: viktor.khmara@student.upjs.sk

${ }^{\ddagger}$ Физический факультет, Ужгородский национальный университет, Ужгород, Украина. E-mail: volodymyr.lazur@uzhnu.edu.ua, oleksandr.reity@uzhnu.edu.ua 


\section{1. ВВЕДЕНИЕ}

Квантово-механическая задача о движении электрона в поле двух кулоновских центров с зарядами $Z_{1}$ и $Z_{2}$, расположенных на расстоянии $R$ друг от друга (так называемая задача $Z_{1} e Z_{2}$ ), исследовалась весьма детально в рамках уравнения Шредингера начиная с конца 20-х гг. XX века. Состояние проблемы и список работ по этой тематике до 1976 г. даны в книге [1]. Интенсивное исследование этой задачи в последние сорок лет объясняется не только появлением мощных ЭВМ и успехами в развитии асимптотических методов решения обыкновенных дифференциальных уравнений, но и потребностями мезомолекулярной физики [2], [3] и теории ион-атомных столкновений [4]. Новые результаты были получены как для само́й задачи о молекулярном ионе водорода $H_{2}^{+}$(см., например, работы [5]-[7] и имеющиеся в них ссылки), так и для задачи двух центров с сильно отличающимися зарядами [8]-[11]. Эта же задача для уравнения Дирака изучалась с помощью асимптотических методов в работах [12], [13]. Вместе с тем задача $Z_{1} e Z_{2}$ исследовалась при малых $R$ в пространствах пониженной [14] и произвольных [15], [16] размерностей.

При решении многих задач, которые возникают в физике медленных атомных столкновений, например при вычислении матричного элемента обменного взаимодействия $\Delta(R)$ атома водорода (или водородоподобного иона) с голым ядром, необходимо знать двухцентровые кулоновские сфероидальные квазирадиальные и квазиугловые волновые функции. Так, для резонансного случая $Z e Z$ упомянутый обменный матричный элемент определялся ранее [17], [18] на основании требования, чтобы вблизи ядер двухцентровая сфероидальная волновая функция электрона переходила в одноцентровую параболическую волновую функцию. На самом деле правильный результат для $\Delta(R)$ можно получить только тогда (это показано в [19]), когда волновые функции нулевого приближения рассматриваются в сфероидальной системе координат. Дело в том, что обменный матричный элемент $\Delta(R)$ определяется асимптотической областью координат электрона, в которой одноцентровые параболические и сфероидальные волновые функции атома водорода существенно отличаются друг от друга. Точнее говоря, на больших расстояниях от ядра вклад в асимптотику кулоновской сфероидальной волновой функции вносит целый набор кулоновских параболических волновых функций. Это обстоятельство затрудняет применение метода эталонного уравнения [1], [8], [9], [11] для определения указанной асимптотики (см., например, [19]).

В качестве подходящего метода расчета волновых функций и всех других необходимых величин в задаче двух кулоновских центров мы предлагаем использовать квазиклассический подход. Этот подход позволяет получить аналитические решения, но он ограничен асимптотически большими межъядерными расстояниями $R$. Эти расстояния должны быть настолько велики, чтобы квантовая проницаемость потенциального барьера, разделяющего атомные частицы, была много меньше единицы. Можно указать большое количество задач [20]-[22], решение которых зависит от этой области межъядерных расстояний.

Структура настоящей статьи следующая. В разделе 2 приведены базовые уравнения для задачи $Z_{1} e Z_{2}$ в сфероидальной системе координат. В разделе 3 с помощью модифицированной теории возмущений получены асимптотические разложения (при больших $R$ ) для двухцентровой квазирадиальной и квазиугловой волновых 
функций с точностью до членов третьего порядка по $1 / R$ включительно. В разделе 4 развивается последовательная схема получения разложений ВКБ для квазиуглового уравнения задачи $Z_{1} e Z_{2}$ в подбарьерной области.

\section{2. ОСНОВНЫЕ УРАВНЕНИЯ}

Движение электрона в поле двух фиксированных ядер с зарядами $Z_{1}$ и $Z_{2}$ описывается следующим уравнением Шредингера $\left(\hbar=e=m_{e}=1\right)$ :

$$
\left(-\frac{1}{2} \Delta-\frac{Z_{1}}{r_{1}}-\frac{Z_{2}}{r_{2}}\right) \Psi(\vec{r}, R)=E(R) \Psi(\vec{r}, R),
$$

где $r_{1}$ и $r_{2}$ - расстояния от электрона до первого и второго ядра, $E(R)$ - энергия электрона, а $R$ - расстояние между ядрами. Уравнение Шредингера (1) допускает разделение переменных в вытянутой сфероидальной системе координат:

$$
\begin{array}{ccc}
\xi=\frac{r_{1}+r_{2}}{R}, & \eta=\frac{r_{1}-r_{2}}{R}, & \phi=\operatorname{arctg} \frac{y}{x}, \\
\xi \in[1 ; \infty), & \eta \in[-1 ; 1], & \phi \in[0 ; 2 \pi),
\end{array}
$$

где $x, y, z$ - декартовы координаты электрона (ось $z$ совпадает с межъядерной осью). Заменяя волновую функцию $\Psi(\vec{r}, R)$ произведением,

$$
\Psi(\vec{r}, R)=\frac{U(\xi, R)}{\sqrt{\xi^{2}-1}} \frac{V(\eta, R)}{\sqrt{1-\eta^{2}}} \frac{e^{ \pm \mathrm{i} m \phi}}{\sqrt{2 \pi}}=\frac{\psi(\xi, \eta, R)}{\sqrt{\left(\xi^{2}-1\right)\left(1-\eta^{2}\right)}} \frac{e^{ \pm \mathrm{i} m \phi}}{\sqrt{2 \pi}},
$$

и используя новые переменные

$$
\mu=\frac{R}{2}(\xi-1), \quad \mu \in[0, \infty), \quad \nu=\frac{R}{2}(1+\eta), \quad \nu \in[0, R],
$$

получаем квазирадиальное и квазиугловое уравнения для $U(\xi, R)$ и $V(\eta, R)$ :

$$
\begin{aligned}
& U^{\prime \prime}(\mu)-\left[\gamma^{2}-\frac{Z_{1}+Z_{2}+\lambda_{1} / R}{\mu}-\frac{Z_{1}+Z_{2}-\lambda_{1} / R}{R+\mu}+\frac{R^{2}\left(m^{2}-1\right)}{4 \mu^{2}(R+\mu)^{2}}\right] U(\mu)=0, \\
& V^{\prime \prime}(\nu)-\left[\gamma^{2}-\frac{Z_{1}-Z_{2}-\lambda_{2} / R}{\nu}+\frac{Z_{1}-Z_{2}+\lambda_{2} / R}{R-\nu}+\frac{R^{2}\left(m^{2}-1\right)}{4 \nu^{2}(R-\nu)^{2}}\right] V(\nu)=0,
\end{aligned}
$$

где $\gamma=(-2 E)^{1 / 2}$. Эти новые функции удовлетворяют следующим граничным условиям:

$$
U(1)=0, \quad U(\xi) \underset{\xi \rightarrow \infty}{\longrightarrow} 0, \quad V( \pm 1)=0 .
$$

В уравнениях (5), (6) параметры $\lambda_{1}$ и $\lambda_{2}$ - постоянные разделения, зависящие от $R$, a $m$ - модуль магнитного квантового числа. Два одномерных уравнения (5) и (6) эквивалентны исходному уравнению Шредингера при условии равенства постоянных разделения:

$$
\lambda_{1}=\lambda_{2}
$$

Если $R$ много больше чем размер электронных оболочек, центрированных на левом ядре, отношения $\mu / R$ и $\nu / R$ являются малыми величинами во внутриатомной области. Этот факт позволяет в этой области применять теорию возмущений к уравнениям (5) и (6) для нахождения как постоянных разделения $\lambda_{1,2}$, так и асимптотик квазирадиальной $U(\mu)$ и квазиугловой $V(\nu)$ волновых функций. 


\section{3. ТЕОРИЯ ВОЗМУЩЕНИЙ И АСИМПТОТИКА КУЛОНОВСКИХ ДВУХЦЕНТРОВЫХ КВАЗИРАДИАЛЬНОЙ И КВАЗИУГЛОВОЙ ВОЛНОВЫХ ФУНКЦИЙ}

При теоретическом описании поведения водородоподобных атомов в поле точечного заряда необходимо в первую очередь построить теорию возмущений при больших межъядерных расстояниях $R$. Несмотря на то что эта задача исследовалась на протяжении многих лет, существующие подходы не позволяют получить простые разложения волновых функций. Так, применение стандартного аппарата теории возмущений Рэлея-Шредингера приводит к появлению бесконечных сумм сложного вида. В связи с этим в ряде работ [23]-[25] были предложены схемы теории возмущений, позволяющие получать аналитические выражения. Одна из таких схем была разработана в работе [25]. Используя ее, мы получим при больших межъядерных расстояниях $R$ асимптотические выражения для двухцентровых квазирадиальной $U(\mu)$ и квазиугловой $V(\nu)$ функций вплоть до членов третьего порядка по $1 / R$ включительно.

Формальная схема дальнейших вычислений включает в себя следующие шаги. Исходный дифференциальный оператор $L$ в уравнениях (5) и (6) представляется в виде суммы: $L=L_{0}+L_{1} / R$. Предельный дифференциальный оператор $L_{0}$ получается из исходного оператора $L$ при $R \rightarrow \infty$. Затем функции $U(\mu)$ и $V(\nu)$ разлагаются в ряды по базисным системам функций $u_{n_{1}}^{(0)}(\mu)$ и $v_{n_{2}}^{(0)}(\nu)$, которые определяются как решения уравнений $L_{0}^{(\mu)} u_{n_{1}}^{(0)}(\mu)=0$ и $L_{0}^{(\nu)} v_{n_{2}}^{(0)}(\nu)=0$ и могут быть выражены в терминах известных специальных функций.

Рассмотрим предельный случай $R \rightarrow \infty$. В этом случае $\lambda$ имеет тот же порядок, что и $R$. Тогда в нулевом приближении (т. е. при $R=\infty$ ) уравнения (5), (6) принимают следующую форму:

$$
\begin{aligned}
& \frac{d^{2} u^{(0)}(\mu)}{d \mu^{2}}-\left[\gamma^{2}-\frac{\varkappa_{1}}{\mu}+\frac{m^{2}-1}{4 \mu^{2}}\right] u^{(0)}(\mu)=0, \\
& \frac{d^{2} v^{(0)}(\nu)}{d \nu^{2}}-\left[\gamma^{2}-\frac{\varkappa_{2}}{\nu}+\frac{m^{2}-1}{4 \nu^{2}}\right] v^{(0)}(\nu)=0,
\end{aligned}
$$

где

$$
\varkappa_{i}=Z_{1} \pm Z_{2} \pm \frac{\lambda^{(0)}}{R} .
$$

Здесь и в дальнейшем индекс $i=1$ и верхний знак соответствуют квазирадиальному уравнению (5), а индекс $i=2$ и нижний знак - квазиугловому уравнению (6).

Решения уравнений (9) и (10), удовлетворяющие граничным условиям (7) при $\mu, \nu \rightarrow 0$, имеют следующий вид:

$$
\begin{aligned}
& u^{(0)}(\mu)=N_{1}^{(0)} e^{-\gamma \mu}(2 \gamma \mu)^{(m+1) / 2} F\left(\frac{m+1}{2}-\frac{\varkappa_{1}}{2 \gamma}, m+1,2 \gamma \mu\right), \\
& v^{(0)}(\nu)=N_{2}^{(0)} e^{-\gamma \nu}(2 \gamma \nu)^{(m+1) / 2} F\left(\frac{m+1}{2}-\frac{\varkappa_{2}}{2 \gamma}, m+1,2 \gamma \nu\right),
\end{aligned}
$$

где $N_{i}^{(0)}$ - нормировочные множители, которые определяются условиями

$$
\int_{0}^{\infty}\left|u^{(0)}(\mu)\right|^{2} d \mu=1, \quad \int_{0}^{\infty}\left|v^{(0)}(\nu)\right|^{2} d \nu=1,
$$


а $F(\alpha, \beta, z)$ - вырожденная гипергеометрическая функция. Чтобы решения (11) и (12) удовлетворяли граничным условиям на бесконечности, параметр $(m+1) / 2-$ $\varkappa_{i} / 2 \gamma$ должен быть равен нулю или целому отрицательному числу,

$$
\frac{m+1}{2}-\frac{\varkappa_{i}}{2 \gamma}=-n_{i}, \quad n_{i}=0,1,2, \ldots
$$

здесь $n_{1}, n_{2}$, и $m$ - параболические квантовые числа. Отсюда для постоянных разделения $\lambda_{n_{i}}^{(0)}(R)$ мы получаем

$$
\lambda_{n_{i}}^{(0)}(R)= \pm R\left[\gamma\left(2 n_{i}+m+1\right)-\left(Z_{1} \pm Z_{2}\right)\right] .
$$

Для нахождения решений при больших, но конечных значениях параметра $R$, следуя работе [25], используем теорию возмущений. В уравнениях (5), (6) будем рассматривать энергию как параметр, а постоянные разделения $\lambda_{i}-$ как собственные значения соответствующих операторов. Тогда вычисление поправок к собственным значениям и собственным функциям производится стандартным образом.

Разложим искомые волновые функции $U(\mu)$ и $V(\nu)$ по системе невозмущенных волновых функций $u_{n_{1}}^{(0)}(\mu)$ и $v_{n_{2}}^{(0)}(\nu)$ :

$$
U(\mu)=\sum_{n_{1}^{\prime}} c_{n_{1}^{\prime}}(R) u_{n_{1}^{\prime}}^{(0)}(\mu), \quad V(\nu)=\sum_{n_{2}^{\prime}} c_{n_{2}^{\prime}}(R) v_{n_{2}^{\prime}}^{(0)}(\nu) .
$$

Подставляя разложение для $U(\mu)$ в $(5)$ и разложение для $V(\nu)$ в $(6)$, умножая полученное квазирадиальное уравнение на $u_{n_{1}}^{(0) *}$, а квазиугловое - на $v_{n_{2}}^{(0) *}$, и интегрируя по переменным $\mu$ и $\nu$, находим

$$
\begin{aligned}
& \left(\lambda_{i}-\lambda_{n_{i}^{\prime}}^{(0)}-\frac{1-m^{2}}{2}\right)\left\langle n_{i}^{\prime}\left|\rho_{i}^{-1}\right| n_{i}^{\prime}\right\rangle c_{n_{i}^{\prime}}= \\
& \quad=\frac{1}{2 \gamma} \sum_{k=0}^{\infty} \frac{(-1)^{i k+1}}{(2 \gamma R)^{k}}\left[Z_{1} \pm Z_{2} \mp \frac{\lambda}{R} \pm(k+3) \frac{1-m^{2}}{4 R}\right] \sum_{n_{i}^{\prime \prime}}\left\langle n_{i}^{\prime}\left|\rho_{i}^{k}\right| n_{i}^{\prime \prime}\right\rangle c_{n_{i}^{\prime \prime}}
\end{aligned}
$$

Здесь $\left\langle n_{i}\left|\rho_{i}^{k}\right| n_{i}^{\prime}\right\rangle$ - матричные элементы операторов $\rho_{1}^{k}=(2 \gamma \mu)^{k}$ и $\rho_{2}^{k}=(2 \gamma \nu)^{k}$ на невозмущенных функциях $u_{n_{1}}^{(0)}(\mu)$ и $v_{n_{2}}^{(0)}(\nu)$ соответственно. Значения этих матричных элементов приведены в приложении А. Соотношение (15) позволяет вычислять поправки любого порядка к собственным значениям и собственным функциям.

Представим постоянную разделения и коэффициенты разложения в (14) в следующей форме:

$$
\lambda_{i}=\lambda_{n_{i}}^{(0)}+\lambda_{n_{i}}^{(1)}+\lambda_{n_{i}}^{(2)}+\lambda_{n_{i}}^{(3)}+\cdots, \quad c_{n_{i}^{\prime}}=c_{n_{i}^{\prime}}^{(0)}+c_{n_{i}^{\prime}}^{(1)}+c_{n_{i}^{\prime}}^{(2)}+c_{n_{i}^{\prime}}^{(3)}+\cdots,
$$

где $\lambda_{n_{i}}^{(k)}$ и $c_{n_{i}^{\prime}}^{(k)}$ являются величинами порядка $R^{-k+1}$ и $R^{-k}$ соответственно.

Для определения поправок к $n$-му собственному значению и $n$-й собственной функции положим $c_{n_{i}}^{(0)}=1$ и $c_{n_{i}^{\prime}}^{(0)}=0$ для $n_{i}^{\prime} \neq n_{i}$. Чтобы найти поправки первого порядка, подставим разложения (16) в уравнение (15), сохранив лишь члены порядка единицы. Полученное уравнение при $n_{i}^{\prime}=n_{i}$ дает

$$
\lambda_{n_{i}}^{(1)}=\frac{1}{2}\left\{\left(2 n_{i}+m+1\right)\left[2 n_{i}+m+1-2 \frac{Z_{1} \pm Z_{2}}{\gamma}\right]+1-m^{2}\right\} .
$$


Из уравнения (15) при $n_{i}^{\prime} \neq n_{i}$ находим коэффициенты:

$$
c_{n_{i}^{\prime}}^{(1)}= \pm \frac{2 n_{i}+m+1-2\left(Z_{1} \pm Z_{2}\right) / \gamma}{4 \gamma R\left(n_{i}-n_{i}^{\prime}\right)} \frac{\left\langle n_{i}^{\prime}\left|\rho_{i}^{0}\right| n_{i}\right\rangle}{\left\langle n_{i}^{\prime}\left|\rho_{i}^{-1}\right| n_{i}^{\prime}\right\rangle}
$$

Коэффициенты $c_{n_{1}}^{(1)}$ и $c_{n_{2}}^{(1)}$ можно определить из условий нормировки волновых функций $u_{n_{1}}(\mu)=u_{n_{1}}^{(0)}(\mu)+u_{n_{1}}^{(1)}(\mu)$ и $v_{n_{2}}(\nu)=v_{n_{2}}^{(0)}(\nu)+v_{n_{2}}^{(1)}(\nu)$, сохранив лишь члены порядка $R^{-1}$ :

$$
c_{n_{i}}^{(1)}= \pm \frac{2 n_{i}+m+1-2\left(Z_{1} \pm Z_{2}\right) / \gamma}{4 \gamma R} .
$$

Для определения поправок второго порядка подставим разложения $\lambda_{i}=\lambda_{n_{i}}^{(0)}+$ $\lambda_{n_{i}}^{(1)}+\lambda_{n_{i}}^{(2)}, c_{n_{i}^{\prime}}=c_{n_{i}^{\prime}}^{(0)}+c_{n_{i}^{\prime}}^{(1)}+c_{n_{i}^{\prime}}^{(2)}$ в уравнение $(15)$ и учтем лишь члены порядка $R^{-1}$. Полученное уравнение при $n_{i}^{\prime}=n_{i}$ дает

$$
\begin{aligned}
\lambda_{n_{i}}^{(2)}= \pm \frac{1}{8 \gamma R}\{ & {\left[2 n_{i}+m+1-2 \frac{Z_{1} \pm Z_{2}}{\gamma}\right]\left[2\left(2 n_{i}+m+1\right) \frac{Z_{1} \pm Z_{2}}{\gamma}-\right.} \\
& \left.\left.-8 n_{i}\left(n_{i}+m+1\right)-(m+1)(m+3)\right]-\left(2 n_{i}+m+1\right)\left(1-m^{2}\right)\right\} .
\end{aligned}
$$

Для случая $n_{i}^{\prime} \neq n_{i}$ из уравнения (15) находим

$$
\begin{aligned}
c_{n_{i}^{\prime}}^{(2)}= \pm & \frac{1}{2 \gamma R\left(n_{i}^{\prime}-n_{i}\right)}\left\{\left(\lambda_{n_{i}}^{(1)}-\frac{1-m^{2}}{2}\right) c_{n_{i}^{\prime}}^{(1)} \mp\right. \\
& \mp \frac{1}{2 \gamma\left\langle n_{i}^{\prime}\left|\rho_{i}^{-1}\right| n_{i}^{\prime}\right\rangle}\left[\frac{1}{R}\left(\lambda_{n_{i}}^{(1)}-\frac{3\left(1-m^{2}\right)}{4}\right)\left\langle n_{i}^{\prime}\left|\rho_{i}^{0}\right| n_{i}\right\rangle+\right. \\
& \left.\left.+\left(\frac{\lambda_{n_{i}}^{(0)}}{R} \mp\left(Z_{1} \pm Z_{2}\right)\right)\left(\sum_{n_{i}^{\prime \prime}}\left\langle n_{i}^{\prime}\left|\rho_{i}^{0}\right| n_{i}^{\prime \prime}\right\rangle c_{n_{i}^{\prime \prime}}^{(1)} \mp \frac{\left\langle n_{i}\left|\rho_{i}^{1}\right| n_{i}^{\prime}\right\rangle}{2 \gamma R}\right)\right]\right\} .
\end{aligned}
$$

Из условий нормировки для волновых функций $u_{n_{1}}=u_{n_{1}}^{(0)}+u_{n_{1}}^{(1)}+u_{n_{1}}^{(2)}$ и $v_{n_{2}}=$ $v_{n_{2}}^{(0)}+v_{n_{2}}^{(1)}+v_{n_{2}}^{(2)}$, сохранив только члены порядка $R^{-2}$, получим

$$
\begin{aligned}
c_{n_{i}}^{(2)}= & \frac{1}{8 \gamma^{2} R^{2}}\left\{\frac{m^{2}-1}{2}-\left[2 n_{i}+m+1-2 \frac{Z_{1} \pm Z_{2}}{\gamma}\right]\left[2 n_{i}+m+3+\frac{4 n_{i}\left(n_{i}+m\right)}{2 n_{i}+m+1}+\right.\right. \\
& \left.\left.+\frac{2 n_{i}+m+1-2\left(Z_{1} \pm Z_{2}\right) / \gamma}{4}\left(2 n_{i}\left(n_{i}+m+1\right)+m+4\right)\right]\right\} .
\end{aligned}
$$


Вычисление поправок более высокого порядка также не вызывает принципиальных трудностей. Для поправок третьего порядка получаем

$$
\begin{aligned}
\lambda_{n_{i}}^{(3)}= & \frac{1}{16 \gamma^{2} R^{2}}\left\{\frac{\left(2 n_{i}+m+1\right)\left[2 n_{i}+m+1-2\left(Z_{1} \pm Z_{2}\right) / \gamma\right]^{3}}{2}-\right. \\
- & \frac{\left[2 n_{i}+m+1-2\left(Z_{1} \pm Z_{2}\right) / \gamma\right]^{2}}{2} \times \\
& \quad \times\left[2\left(2 n_{i}+m+1\right) \frac{Z_{1} \pm Z_{2}}{\gamma}-36 n_{i}\left(n_{i}+m+1\right)-(5 m+13)(m+1)\right]+ \\
+ & \left(2 n_{i}+m+1\right)\left[2 n_{i}+m+1-2 \frac{Z_{1} \pm Z_{2}}{\gamma}\right]\left[2\left(2 n_{i}+m+1\right) \frac{Z_{1} \pm Z_{2}}{\gamma}+2\left(3-m^{2}\right)\right]+ \\
+ & \left.\left(1-m^{2}\right)\left[8 n_{i}\left(n_{i}+m+1\right)+(m+1)(m+3)\right]\right\},
\end{aligned}
$$

$$
\begin{aligned}
c_{n_{i}^{\prime}}^{(3)} & = \pm \frac{1}{2 \gamma R\left(n_{i}-n_{i}^{\prime}\right)}\left\{-\lambda_{n_{i}}^{(2)} c_{n_{i}^{\prime}}^{(1)}-\left(\lambda_{n_{i}}^{(1)}-\frac{1-m^{2}}{2}\right) c_{n_{i}^{\prime}}^{(2)} \pm\right. \\
& \pm \frac{1}{\left\langle n_{i}^{\prime}\left|\rho_{i}^{-1}\right| n_{i}^{\prime}\right\rangle}\left[\frac{\lambda_{n_{i}}^{(2)}}{2 \gamma R}\left\langle n_{i}\left|\rho_{i}^{0}\right| n_{i}^{\prime}\right\rangle \mp \frac{Z_{1} \pm Z_{2} \mp \lambda_{n_{i}}^{(0)} / R}{2 \gamma} \sum_{n_{i}^{\prime \prime}} c_{n_{i}^{\prime \prime}}^{(2)}\left\langle n_{i}^{\prime \prime}\left|\rho_{i}^{0}\right| n_{i}^{\prime}\right\rangle+\right. \\
& +\left(\frac{\lambda_{n_{i}}^{(1)}}{2 \gamma R}-\frac{3\left(1-m^{2}\right)}{8 \gamma R}\right) \sum_{n_{i}^{\prime \prime}}\left\langle n_{i}^{\prime}\left|\rho_{i}^{0}\right| n_{i}^{\prime \prime}\right\rangle c_{n_{i}^{\prime \prime}}^{(1)}+\frac{Z_{1} \pm Z_{2} \mp \lambda_{n_{i}}^{(0)} / R}{4 \gamma^{2} R} \sum_{n_{i}^{\prime \prime}} c_{n_{i}^{\prime \prime}}^{(1)}\left\langle n_{i}^{\prime \prime}\left|\rho_{i}^{1}\right| n_{i}^{\prime}\right\rangle \mp \\
& \left.\left.\mp \frac{\lambda_{n_{i}}^{(1)}+m^{2}-1}{4 \gamma^{2} R^{2}}\left\langle n_{i}\left|\rho_{i}^{1}\right| n_{i}^{\prime}\right\rangle \mp \frac{Z_{1} \pm Z_{2} \mp \lambda_{n_{i}}^{(0)} / R}{8 \gamma^{3} R^{2}}\left\langle n_{i}\left|\rho_{i}^{2}\right| n_{i}^{\prime}\right\rangle\right]\right\},
\end{aligned}
$$

$$
\begin{aligned}
& c_{n_{i}}^{(3)}= \pm \frac{m^{2}-1}{32 \gamma^{3} R^{3}}\left(2 n_{i}+m+1-4\left\langle n_{i}\left|\rho_{i}^{1}\right| n_{i}\right\rangle\right) \pm \\
& \quad \pm \frac{2 n_{i}+m+1-2\left(Z_{1} \pm Z_{2}\right) / \gamma}{64 \gamma^{3} R^{3}}\left[\left(33-m^{2}\right)\left(2 n_{i}\left(n_{i}+m+1\right)+m+4\right)+56\right] \pm \\
& \quad \pm \frac{\left[2 n_{i}+m+1-2\left(Z_{1} \pm Z_{2}\right) / \gamma\right]^{2}}{32 \gamma^{3} R^{3}}\left[\left(2 n_{i}+m+1\right)\left(2 n_{i}\left(n_{i}+m+1\right)+m-1\right)+\right. \\
& \left.\quad+10\left\langle n_{i}\left|\rho_{i}^{1}\right| n_{i}\right\rangle\right] \pm \frac{\left[2 n_{i}+m+1-2\left(Z_{1} \pm Z_{2}\right) / \gamma\right]^{3}}{128 \gamma^{3} R^{3}}\left[6 n_{i}\left(n_{i}+m+1\right)+3 m+10\right] .
\end{aligned}
$$

Параметр $\gamma$ можно определить из формулы (8). Принимая во внимание равенство $n_{1}+n_{2}+m+1=n$, получим

$$
\gamma=\gamma_{0}+\frac{\gamma_{1}}{R}+\frac{\gamma_{2}}{R^{2}}+\frac{\gamma_{3}}{R^{3}}+\cdots
$$

где

$$
\begin{gathered}
\gamma_{0}=\frac{Z_{1}}{n}, \quad \gamma_{1}=\frac{n Z_{2}}{Z_{1}}, \quad \gamma_{2}=-\frac{n^{2} Z_{2}}{2 Z_{1}^{3}}\left[3\left(n_{1}-n_{2}\right) Z_{1}+n Z_{2}\right], \\
\gamma_{3}=\frac{Z_{2} n^{3}}{2 Z_{1}^{5}}\left\{Z_{1}^{2}\left[6\left(n_{1}-n_{2}\right)^{2}+1-n^{2}\right]+3 Z_{1} Z_{2} n\left(n_{1}-n_{2}\right)+Z_{2}^{2} n^{2}\right\} .
\end{gathered}
$$

Выражения $E=-\gamma^{2} / 2$ и (26) дают хорошо известное [1] разложение по мультиполям энергии электростатического взаимодействия атома $e Z_{1}$ с удаленным точечным 
зарядом $Z_{2}$. Отметим также, что в формуле (4.60) монографии [1] выражение для $\gamma_{3}$ содержит опечатку.

Переход от левого центра $Z_{1}$ к правому $Z_{2}$ в приведенных выше формулах осуществляется заменами $Z_{1} \leftrightarrow Z_{2}, n_{i} \rightarrow n_{i}^{\prime}, \nu \rightarrow R-\nu, \phi \rightarrow-\phi$. Параболические квантовые числа $n_{1}^{\prime}, n_{2}^{\prime}$ на правом центре удовлетворяют условию $n_{1}^{\prime}+n_{2}^{\prime}+m+1=n^{\prime}$.

Таким образом, мы показали, что использование модифицированной схемы теории возмущений [25] позволяет получать простые разложения по степеням $1 / R$ для волновой функции водородоподобного атома в поле удаленного точечного заряда. При этом функция $\psi$ в формуле (3) с точностью до членов порядка $R^{-3}$ включительно принимает вид

$$
\psi^{\text {pert }}(\mu, \nu)=C U^{\text {pert }}(\mu) V^{\text {pert }}(\nu)
$$

где

$$
\begin{gathered}
U^{\text {pert }}=f_{n_{1}}^{(0)}(\mu)+\sum_{p=1}^{3} \sum_{k=-p}^{p} c_{n_{1}+k}^{(p)} f_{n_{1}+k}^{(0)}(\mu), \\
V^{\text {pert }}=f_{n_{2}}^{(0)}(\nu)+\sum_{p=1}^{3} \sum_{k=-p}^{p} c_{n_{2}+k}^{(p)} f_{n_{2}+k}^{(0)}(\nu), \\
f_{n_{i}}^{(0)}(x)=\left(\frac{\left(n_{i}+m\right) !}{n_{i} !(m !)^{2}\left(2 n_{i}+m+1\right)}\right)^{1 / 2} e^{-\gamma x}(2 \gamma x)^{(m+1) / 2} F\left(-n_{i}, m+1,2 \gamma x\right),
\end{gathered}
$$

а все коэффициенты $c_{n_{i}+k}^{(p)}$ для $p=1,2,3$ были найдены выше.

Нормируем теперь полную волновую функцию $\Psi(\vec{r}, R)$, заданную формулами (3), (28), с точностью до членов $R^{-3}$ включительно. Для постоянной нормировки $C$ окончательно получаем

$$
\begin{aligned}
C(R)=\frac{4 \gamma^{1 / 2}}{R} & \left\{\sum _ { i = 1 } ^ { 2 } \left[\left\langle n_{i}\left|\rho_{i}^{-1}\right| n_{i}\right\rangle\left(1+2 c_{n_{i}}^{(1)}+2 c_{n_{i}}^{(2)}+2 c_{n_{i}}^{(3)}\right)+\right.\right. \\
& \left.+\sum_{k=-1}^{1}\left(\left|c_{n_{i}+k}^{(1)}\right|^{2}+2 c_{n_{i}+k}^{(1)} c_{n_{i}+k}^{(2)}\right)\left\langle n_{i}+k\left|\rho_{i}^{-1}\right| n_{i}+k\right\rangle\right]+ \\
& \left.+\frac{1}{4 \gamma^{2} R^{2}} \sum_{i=1}^{2}\left\langle n_{i}\left|\rho_{i}^{1}\right| n_{i}\right\rangle\left(1-2 c_{n_{i}}^{(1)}\right)-\frac{\left\langle n_{1}\left|\rho_{1}^{2}\right| n_{1}\right\rangle-\left\langle n_{2}\left|\rho_{2}^{2}\right| n_{2}\right\rangle}{8 \gamma^{3} R^{3}}\right\}^{-1 / 2} .
\end{aligned}
$$

Полученные функции (3), (28), (29) описывают поведение электронов в основной области распределения электронной плотности.

Следующей нашей задачей будет нахождение двухцентровых волновых функций в межъядерной области, когда электрон находится далеко от обоих кулоновских центров (ядер). Ранее в работе [26] решение квазиуглового уравнения (6) в указанной области удалось получить лишь в предельном случае $R \gg 2 n^{2} / Z_{1}$. Однако используемый в [26] известный асимптотический метод Ландау-Херринга [27] имеет ограниченную область применимости вследствие приближений, которые используются при вычислении так называемых поправочных функций. Поэтому в следующем разделе для нахождения квазиугловой функции $V(\nu)$ под потенциальным барьером 


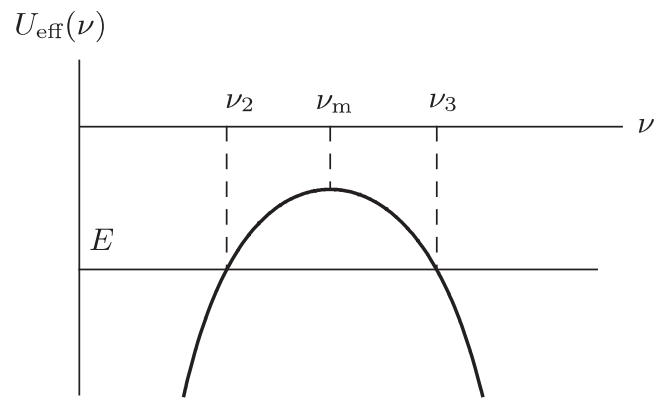

a

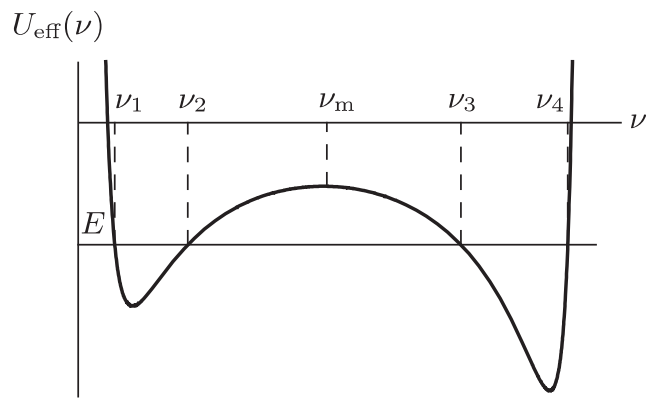

б

Рис. 1. Эффективный потенциал $U_{\mathrm{eff}}(\nu)$ квазиуглового уравнения $(31)$ при $m=0,1$ (а) и при $m>1$ (б).

мы воспользуемся квазиклассическим приближением (методом ВКБ), которое может применяться и в области не слишком больших межъядерных расстояний. При этом условие квазиклассичности движения импользуется лишь по квазиугловой переменной $\nu$ с сохранением пертурбативного решения задачи $Z_{1} e Z_{2}$ по квазирадиальной переменной $\mu$, и это позволяет выразить матричный элемент обменного взаимодействия [22], характеризующего процесс перезарядки водородоподобных атомов на голых ядрах, через квазиклассическую проницаемость потенциального барьера, разделяющего потенциальные ямы (см. рис. 1) в квазиугловом уравнении (6).

\section{4. КВАЗИКЛАССИЧЕСКИЕ РЕШЕНИЯ КВАЗИУГЛОВОГО УРАВНЕНИЯ В МЕЖЪЯДЕРНОЙ ОБЛАСТИ}

Хотя теория возмущений позволяет решить принципиальные вопросы, связанные с разложениями собственных значений $\lambda_{1,2}(R)$ и собственных функций $U(\mu)$ и $V(\nu)$ в ряды по степеням $1 / R$, нахождение последующих членов в этих разложениях вызывает быстро нарастающие вычислительные трудности. Решения уравнений (5), (6) при больших $R$ можно представить в достаточно простом и компактном виде, воспользовавшись методом ВКБ (или квазиклассическим приближением), который был развит и получил известность как один из наиболее эффективных методов приближенного решения задач квантовой механики (см., например, труды [28]-[31]). Метод ВКБ позволяет получить равномерные оценки для собственных функций при произвольно больших межъядерных расстояниях $R$. Другим достоинством квазиклассических асимптотик является их простота. Кроме того, в отличие от теории возмущений, квазиклассическое приближение не связано с малостью взаимодействия и поэтому имеет более широкую область применимости, позволяя исследовать качественные закономерности в поведении и свойствах квантово-механических систем. Ниже мы построим квазиклассическое решение квазиуглового уравнения в классически запрещенной области.

Перепишем квазиугловое уравнение (6) в форме одномерного уравнения Шредингера

$$
V^{\prime \prime}-\frac{q^{2}}{\hbar^{2}} V=0
$$


где $q=\sqrt{2\left(U_{\text {eff }}-E\right)}$, а функция $U_{\text {eff }}(\nu)$ играет роль эффективной потенциальной энергии в квазиугловом уравнении:

$$
U_{\text {eff }}(\nu)=-\frac{\widetilde{Z}_{1}}{\nu}-\frac{\widetilde{Z}_{2}}{R-\nu}+\frac{\hbar^{2}\left(m^{2}-1\right)}{8 \nu^{2}(1-\nu / R)^{2}}, \quad \widetilde{Z}_{1,2}=\frac{ \pm\left(Z_{1}-Z_{2}\right)-\lambda / R}{2} .
$$

Здесь восстановлена в явном виде зависимость от постоянной Планка $ћ$ и введены следующие обозначения (см. рис. 1$): \nu_{i}(i=\overline{1,4})$ - точки поворота, а $\nu_{\mathrm{m}}$ - та точка, в которой эффективный потенциал $U_{\text {eff }}$ достигает максимума. При этом величина $q$ вещественная и с точностью до мнимой единицы і совпадает с квазиугловым импульсом классической частицы, $q(\nu)>0$ при $\nu_{2}<\nu<\nu_{3}$. Представим решение уравнения (31) в виде формального ряда по степеням $\hbar$ :

$$
V^{\mathrm{semi}}=e^{S / \hbar}, \quad S=\sum_{k=-1}^{\infty} \hbar^{k} S_{k} .
$$

Подставляя это выражение в (31) и приравнивая к нулю коэффициенты при одинаковых степенях $\hbar$, получаем рекуррентную систему дифференциальных уравнений первого порядка для неизвестных функций $S_{k}(\nu)$ :

$$
\begin{aligned}
& \left(S_{-1}^{\prime}\right)^{2}=q^{2}, \\
& 2 S_{-1}^{\prime} S_{0}^{\prime}+S_{-1}^{\prime \prime}=0, \\
& 2 S_{-1}^{\prime} S_{k}^{\prime}+S_{k-1}^{\prime \prime}+\sum_{j=0}^{k-1} S_{j}^{\prime} S_{k-j-1}^{\prime}=0, \quad k=1,2, \ldots .
\end{aligned}
$$

В качестве решения уравнения (33) выбираем функцию

$$
S_{-1}(\nu)=-\int_{\nu_{2}}^{\nu} q\left(\nu^{\prime}\right) d \nu^{\prime}
$$

соответствующую экспоненциальному затуханию функции $V^{\mathrm{semi}}(\nu)$ вглубь подбарьерной области. Уравнение для $S_{0}$ решается в замкнутом виде: $S_{0}=\ln \left(C_{0} / \sqrt{q}\right)$. Решения всех последующих уравнений рекуррентной системы (35) выражаются в квадратурах:

$$
S_{k}=\int \frac{1}{2 q}\left(S_{k-1}^{\prime \prime}+\sum_{j=0}^{k-1} S_{j}^{\prime} S_{k-j-1}^{\prime}\right) d \nu+C_{k}, \quad C_{k}=\text { const }, \quad k=1,2, \ldots .
$$

Для дальнейшего рассмотрения удобно ввести две области изменения переменной $\nu$ : $0 \leqslant \nu \ll \nu_{\mathrm{m}}$ и $\nu_{2} \ll \nu \ll \nu_{3}$. В первой области справедливы полученные в разделе 3 формулы теории возмущений (29), а чтобы найти квазиугловую функцию $V(\nu)$, определяющую поведение электрона в подбарьерной области $\nu_{2}<\nu<\nu_{3}$, мы воспользовались квазиклассическим подходом. Важно отметить, что там, где области применимости этих двух методов перекрываются, их результаты совпадают. Это позволяет определить постоянные интегрирования $C_{k}$ из условия сшивки решений $V^{\text {semi }}(32)$ и $V^{\text {pert }}(29)$ :

$$
V^{\mathrm{semi}}(\nu) \underset{\nu_{2} \ll \nu \ll \nu_{\mathrm{m}}}{\longrightarrow} V^{\mathrm{as}}(\nu) .
$$


Для выполнения условия (38) необходимо, чтобы межъядерное расстояние было много больше того, при котором исчезает потенциальный барьер в квазиугловом уравнении (31), т. е.

$$
R \gg R_{0}=\frac{1}{2 \gamma^{2}}\left[2\left(\widetilde{Z}_{1}+\widetilde{Z}_{2}\right)+\sqrt{4 \widetilde{Z}_{1}^{2}+\gamma^{2}\left(1-m^{2}\right)}+\sqrt{4 \widetilde{Z}_{2}^{2}+\gamma^{2}\left(1-m^{2}\right)}\right] .
$$

При выполнении этого условия поляризационное смещение энергии электрона (см. соотношения $(26),(27))$ мало по сравнению с энергией связи электрона в изолированном атоме $e Z_{1}$.

Таким образом, решение квазиуглового уравнения (31) в квазиклассическом приближении, удовлетворяющее граничному условию (38), имеет вид (здесь и далее мы полагаем $\hbar=1)$

$$
V^{\mathrm{semi}}=\frac{C_{0}}{\sqrt{q}} \exp \left[-\int_{\nu_{2}}^{\nu} q\left(\nu^{\prime}\right) d \nu^{\prime}+S_{1}+S_{2}\right]
$$

где квазиклассические поправки $S_{1}$ и $S_{2}$ определяются формулами

$$
\begin{aligned}
S_{1}= & -\frac{\widetilde{Z}_{1}}{4 \gamma^{3} \nu^{2}}\left(1+\frac{17 \widetilde{Z}_{1}}{6 \gamma^{2} \nu}\right)+\frac{\widetilde{Z}_{2}}{4 \gamma^{3}(R-\nu)^{2}}\left(1+\frac{17 \widetilde{Z}_{2}}{6 \gamma^{2}(R-\nu)}\right)+ \\
& +\frac{m^{2}-1}{16 \gamma^{3}}\left(\frac{1}{\nu^{3}}+\frac{1}{\nu^{2} R}-\frac{1}{R(R-\nu)^{2}}-\frac{1}{(R-\nu)^{3}}\right)+\frac{\widetilde{Z}_{1} \widetilde{Z}_{2}}{2 \gamma^{5} R^{3}} \ln \frac{\nu}{R-\nu}+ \\
& +\frac{\widetilde{Z}_{1} \widetilde{Z}_{2}}{4 \gamma^{5} R}\left(\frac{3}{(R-\nu)^{2}}-\frac{3}{\nu^{2}}+\frac{1}{R}\left[\frac{1}{R-\nu}-\frac{1}{\nu}\right]\right)+C_{1}, \\
S_{2}= & \frac{\widetilde{Z}_{1}}{4 \gamma^{4} \nu^{3}}+\frac{\widetilde{Z}_{2}}{4 \gamma^{4}(R-\nu)^{3}}+C_{2},
\end{aligned}
$$

а выражения для постоянных интегрирования $C_{0}, C_{1}$ и $C_{2}$ приведены в приложении Б. Аналогичным образом определяются и следующие члены $S_{3}, S_{4}, \ldots$ в разложении (32). Мы, однако, ограничимся найденными членами $S_{-1}, S_{0}, S_{1}$ и $S_{2}$, так как учет поправок более высокого порядка по $ћ$ обычно не улучшает согласие метода ВКБ с точным решением. Причина этого, как известно [27]-[31], заключается в том, что формальный ряд по степеням $\hbar$ является не сходящимся, а только асимптотическим.

Таким образом, полученная формула (40) определяет квазиклассическую асимптотику решений квазиуглового уравнения (31) при $\hbar \rightarrow 0$ и справедлива в подбарьерной области $\nu_{2}<\nu<\nu_{3}$.

Хотя формулы (40)-(42) существенно отличаются от формул теории возмущений (29), их применение к конкретным задачам не встречает затруднений, так как величины $S_{k}$, входящие в $V^{\text {semi }}(31)$, определены через квадратуры (37).

Окончательное выражение для двухцентровой волновой функции $\Psi$ системы $Z_{1} e Z_{2}$ имеет вид (3), где

$$
\psi(\mu, \nu)=C(R) U^{\text {pert }}(\mu) V^{\text {semi }}(\nu),
$$

а постоянная нормировки $C(R)$ по-прежнему дается формулой (30). 


\section{5. ЗАКЛЮЧЕНИЕ}

С помощью модифицированной теории возмущений получены асимптотические выражения для двухцентровых квазирадиальной и квазиугловой волновых функций при больших межъядерных расстояниях $R$. Показано, что в каждом порядке по $R^{-1}$ поправки к волновым функциям выражаются через конечное число кулоновских функций с модифицированным зарядом. Для первой, второй и третьей поправок к квазирадиальной и квазиугловой функциям получены простые аналитические выражения, которые необходимы для постановки граничных условий в адиабатическом представлении задачи трех тел [1].

В рамках квазиклассического подхода найдено аналитическое выражение для решений квазиуглового уравнения (6) в области подбарьерного движения электрона. Будучи обычно справедливым для высоковозбужденных состояний, этот подход дает точность вычисления уровней энергии порядка $1 / n$ (где $n$ - главное квантовое число состояния электрона в изолированном атоме $e Z_{1}$ ). Поэтому для исследования низколежащих состояний необходимо использовать высшие порядки квазиклассического приближения, нахождение которых с помощью развитой в настоящей работе рекуррентной схемы не вызывает принципиальных затруднений. Полученные асимптотические выражения для квазирадиальных и квазиугловых функций будут использованы в наших дальнейших исследованиях при расчете матричного элемента обменного взаимодействия, характеризующего процесс перезарядки водородоподобного атома (иона) на многозарядном ионе.

Имеются две области расстояний между кулоновскими центрами, где решения квазиуглового уравнения (6) различным образом ведут себя в зависимости от изменения межъядерного расстояния $R$ и квазиугловой переменной $\nu$. Так, в области $R \gg 2 n^{2} / Z_{1}$ квазиклассическое решение $(40)$ для $V(\nu)$ переходит в предельное выражение (29), которое получено в рамках модифицированной теории возмущений. В области $R_{0}<R<2 n^{2} / Z_{1}$ теория возмущений неприменима, так как она не учитывает точную форму потенциального барьера, образованного двумя кулоновскими центрами. В то же время расчеты показали, что использованный в настоящей работе квазиклассический метод правильно описывает поведение электрона как при промежуточных межъядерных расстояниях $R_{0}<R<2 n^{2} / Z_{1}$, так и в асимптотическом пределе $R \gg 2 n^{2} / Z_{1}$.

\section{ПРИЛОЖЕНИЕ А}

\section{Матричные элементы}

Приведем выражения для матричных элементов операторов $\rho_{i}^{k}$ с $k=-1,0,1,2$ и $i=1,2$, где $\rho_{1}=2 \gamma \mu, \rho_{2}=2 \gamma \nu$. Диагональные матричные элементы имеют вид

$$
\begin{aligned}
\left\langle n_{i}\left|\rho_{i}^{-1}\right| n_{i}\right\rangle & =\frac{1}{2 n_{i}+m+1}, \quad\left\langle n_{i}\left|\rho_{i}^{0}\right| n_{i}\right\rangle=1, \\
\left\langle n_{i}\left|\rho_{i}^{1}\right| n_{i}\right\rangle & =\frac{6 n_{i}\left(n_{i}+m+1\right)+(m+1)(m+2)}{2 n_{i}+m+1}, \\
\left\langle n_{i}\left|\rho_{i}^{2}\right| n_{i}\right\rangle & =\frac{1}{2 n_{i}+m+1}\left[(m+1)(m+2)(m+3)+12 n_{i}(m+2)(m+3)+\right. \\
& \left.+30 n_{i}\left(n_{i}-1\right)(m+3)+20 n_{i}\left(n_{i}-1\right)\left(n_{i}-2\right)\right] .
\end{aligned}
$$


Недиагональные матричные элементы имеют вид

$$
\begin{aligned}
& \left\langle n_{i}-1\left|\rho_{i}^{0}\right| n_{i}\right\rangle=\left\langle n_{i}\left|\rho_{i}^{0}\right| n_{i}-1\right\rangle=-\left(\frac{n_{i}\left(n_{i}+m\right)}{\left(2 n_{i}+m+1\right)\left(2 n_{i}+m-1\right)}\right)^{1 / 2}, \\
& \left\langle n_{i}+1\left|\rho_{i}^{0}\right| n_{i}\right\rangle=\left\langle n_{i}\left|\rho_{i}^{0}\right| n_{i}+1\right\rangle=-\left(\frac{\left(n_{i}+1\right)\left(n_{i}+m+1\right)}{\left(2 n_{i}+m+1\right)\left(2 n_{i}+m+3\right)}\right)^{1 / 2}, \\
& \left\langle n_{i}-1\left|\rho_{i}^{1}\right| n_{i}\right\rangle=\left\langle n_{i}\left|\rho_{i}^{1}\right| n_{i}-1\right\rangle=-2\left(\frac{n_{i}\left(n_{i}+m\right)\left(2 n_{i}+m\right)^{2}}{\left(2 n_{i}+m+1\right)\left(2 n_{i}+m-1\right)}\right)^{1 / 2}, \\
& \left\langle n_{i}+1\left|\rho_{i}^{1}\right| n_{i}\right\rangle=\left\langle n_{i}\left|\rho_{i}^{1}\right| n_{i}+1\right\rangle=-2\left(\frac{\left(n_{i}+1\right)\left(n_{i}+m+1\right)\left(2 n_{i}+m+2\right)^{2}}{\left(2 n_{i}+m+1\right)\left(2 n_{i}+m+3\right)}\right)^{1 / 2}, \\
& \left\langle n_{i}-2\left|\rho_{i}^{1}\right| n_{i}\right\rangle=\left\langle n_{i}\left|\rho_{i}^{1}\right| n_{i}-2\right\rangle=\left(\frac{n_{i}\left(n_{i}-1\right)\left(n_{i}+m\right)\left(n_{i}+m-1\right)}{\left(2 n_{i}+m+1\right)\left(2 n_{i}+m-3\right)}\right)^{1 / 2}, \\
& \left\langle n_{i}+2\left|\rho_{i}^{1}\right| n_{i}\right\rangle=\left\langle n_{i}\left|\rho_{i}^{1}\right| n_{i}+2\right\rangle=\left(\frac{\left(n_{i}+1\right)\left(n_{i}+2\right)\left(n_{i}+m+1\right)\left(n_{i}+m+2\right)}{\left(2 n_{i}+m+1\right)\left(2 n_{i}+m+5\right)}\right)^{1 / 2}, \\
& \left\langle n_{i}-1\left|\rho_{i}^{2}\right| n_{i}\right\rangle=\left\langle n_{i}\left|\rho_{i}^{2}\right| n_{i}-1\right\rangle=-3\left(\frac{n_{i}\left(n_{i}+m\right)}{\left(2 n_{i}+m+1\right)\left(2 n_{i}+m-1\right)}\right)^{1 / 2} \times \\
& \times\left[5 n_{i}\left(n_{i}+m\right)+m^{2}+1\right] \text {, } \\
& \left\langle n_{i}+1\left|\rho_{i}^{2}\right| n_{i}\right\rangle=\left\langle n_{i}\left|\rho_{i}^{2}\right| n_{i}+1\right\rangle=-3\left(\frac{\left(n_{i}+1\right)\left(n_{i}+m+1\right)}{\left(2 n_{i}+m+1\right)\left(2 n_{i}+m+3\right)}\right)^{1 / 2} \times \\
& \times\left[5 n_{i}\left(n_{i}+m+1\right)+(m+2)(m+3)\right], \\
& \left\langle n_{i}-2\left|\rho_{i}^{2}\right| n_{i}\right\rangle=\left\langle n_{i}\left|\rho_{i}^{2}\right| n_{i}-2\right\rangle=3\left(\frac{n_{i}\left(n_{i}-1\right)\left(n_{i}+m\right)\left(n_{i}+m-1\right)}{\left(2 n_{i}+m+1\right)\left(2 n_{i}+m-3\right)}\right)^{1 / 2}\left(2 n_{i}+m-1\right), \\
& \left\langle n_{i}+2\left|\rho_{i}^{2}\right| n_{i}\right\rangle=\left\langle n_{i}\left|\rho_{i}^{2}\right| n_{i}+2\right\rangle=3\left(\frac{\left(n_{i}+1\right)\left(n_{i}+2\right)\left(n_{i}+m+1\right)\left(n_{i}+m+2\right)}{\left(2 n_{i}+m+1\right)\left(2 n_{i}+m+5\right)}\right)^{1 / 2} \times \\
& \times\left(2 n_{i}+m+3\right), \\
& \left\langle n_{i}-3\left|\rho_{i}^{2}\right| n_{i}\right\rangle=\left\langle n_{i}\left|\rho_{i}^{2}\right| n_{i}-3\right\rangle= \\
& =-\left(\frac{n_{i}\left(n_{i}-1\right)\left(n_{i}-2\right)\left(n_{i}+m\right)\left(n_{i}+m-1\right)\left(n_{i}+m-2\right)}{\left(2 n_{i}+m+1\right)\left(2 n_{i}+m-5\right)}\right)^{1 / 2}, \\
& \left\langle n_{i}+3\left|\rho_{i}^{2}\right| n_{i}\right\rangle=\left\langle n_{i}\left|\rho_{i}^{2}\right| n_{i}+3\right\rangle= \\
& =-\left(\frac{\left(n_{i}+1\right)\left(n_{i}+2\right)\left(n_{i}+3\right)\left(n_{i}+m+1\right)\left(n_{i}+m+2\right)\left(n_{i}+m+3\right)}{\left(2 n_{i}+m+1\right)\left(2 n_{i}+m+7\right)}\right)^{1 / 2} .
\end{aligned}
$$

Отметим также, что в работе [25] есть ошибки в формулах для матричных элементов $\left\langle n_{i}\left|\rho_{i}^{2}\right| n_{i}\right\rangle$ и $\left\langle n_{i}+1\left|\rho_{i}^{1}\right| n_{i}\right\rangle$.

ПРИЛОЖКНИЕ Б

\section{Постоянные интегрирования}

Постоянные интегрирования $C_{0}, C_{1}, C_{2}$ равны

$$
C_{0}=(-1)^{n_{2}} e^{-\widetilde{Z}_{1} / \gamma} Q_{+} Q_{-}, \quad Q_{ \pm}=\left(\frac{\widetilde{Z}_{1}}{\gamma} \pm \frac{\sqrt{m^{2}-1}}{2}\right)^{\left\{n_{2}+1 /[1 \pm \sqrt{(m-1) /(m+1)}]\right\} / 2},
$$


И

$$
\begin{aligned}
& C_{1}=-\frac{2 n_{2}+m+1-2\left(Z_{1}-Z_{2}\right) / \gamma}{2 \gamma R}+ \\
& +\frac{\left(Z_{1}-Z_{2}\right)^{2}}{8 \gamma^{4} R^{2}}\left[\frac{\left(m^{2}-1\right)\left(2 n_{2}+m+1\right)}{2 n_{2}\left(n_{2}+m+1\right)+m+1}+2\left(2 n_{2}+m-3\right)\right]+ \\
& +\frac{Z_{1}-Z_{2}}{4 \gamma^{3} R^{2}}\left[2\left\langle n_{2}\left|\rho_{2}^{1}\right| n_{2}\right\rangle-2 n_{2}\left(2 n_{2}+2 m-3\right)-m^{2}+3 m+5\right]+ \\
& +\frac{1}{8 \gamma^{2} R^{2}}\left[2 n_{2}\left(n_{2}+1\right)\left(2 n_{2}-11\right)+m^{2}\left(2 n_{2}-3\right)+m\left(6 n_{2}\left(n_{2}-3\right)-11\right)-8\right] \text {, } \\
& C_{2}=-\frac{\left(Z_{1}-Z_{2}\right)^{3}}{24 \gamma^{6} R^{3}\left[2 n_{2}\left(n_{2}+m+1\right)+m+1\right]^{2}} \times \\
& \times\left\{2 n_{2}^{2}(m+1)\left(98 n_{2} m-26 n_{2}+47 m^{2}+36 m-75\right)+\right. \\
& +(m+1)^{2}\left[m\left(50 n_{2}+22 n_{2} m+2 m^{2}+7 m+8\right)-100 n_{2}-29\right]+ \\
& \left.+8 n_{2}^{4}(25 m+9)+80 n_{2}^{5}\right\}+ \\
& +\frac{\left(Z_{1}-Z_{2}\right)^{2}}{24 \gamma^{5} R^{3}\left[\left(2 n_{2}\left(n_{2}+m+1\right)+m+1\right]\left(2 n_{2}+m+1\right)\right.} \times \\
& \times\left\{(1+m)^{2}\left[9 m\left(m^{2}+3 m-9\right)-152\right]+\right. \\
& +2 n_{2}(m+1)\left(-385-386 m+27 m^{2}+45 m^{3}-771 n_{2}-252 m n_{2}+180 m^{2} n_{2}\right)+ \\
& \left.+144 n_{2}^{4}\left(2 n_{2}+5 m-2\right)+4 n_{2}^{3}(6 m-11)(30 m+31)\right\}- \\
& -\frac{Z_{1}-Z_{2}}{48 \gamma^{4} R^{3}}\left\{21 m^{3}-114 m^{2}-413 m-254+168 n_{2}^{3}-n_{2}^{2}\left(6 m^{2}-252 m+558\right)-\right. \\
& \left.-n_{2}\left(6 m^{3}-126 m^{2}+558 m+826\right)\right\}+ \\
& +\frac{1}{96 \gamma^{3} R^{3}}\left\{128 n_{2}^{4}+12\left(m^{2}-1\right)\left\langle n_{2}\left|\rho_{2}^{1}\right| n_{2}\right\rangle-4 n_{2}^{3}\left[3 m^{2}-64 m+121\right]-\right. \\
& -6 n_{2}^{2}\left[m\left(3 m^{2}-35 m+121\right)+183\right]-2 n_{2} m\left[m\left(3 m^{2}-11 m+180\right)+549\right]- \\
& \left.-662 n_{2}-133-m\left[m\left(3 m^{2}+59 m+254\right)+331\right]\right\}+\frac{\widetilde{Z}_{1} \widetilde{Z}_{2}}{2 \gamma^{5} R^{3}} \ln (2 \gamma R)- \\
& -\frac{2 n_{2}+m+1-2\left(Z_{1}-Z_{2}\right) / \gamma}{32 \gamma^{3} R^{3}}\left[\left(2 n_{2}\left(n_{2}+m+1\right)+m\right)\left(33-m^{2}\right)+56\right] .
\end{aligned}
$$

\section{Список литературы}

[1] И. В. Комаров, Л. И. Пономарев, С. Ю. Славянов, Сфероидальные и кулоновские сфероидальные функиии, Наука, М., 1976.

[2] Л. И. Меньшиков, Л.Н. Сомов, "Современное состояние мюонного катализа ядерных реакций синтеза", УФН, 160:8 (1990), 47-103.

[3] С. С. Герштейн, Ю. В. Петров, Л. И. Пономарев, "Мюонный катализ и ядерный бридинг", УФН, 160:8 (1990), 3-46.

[4] R. K. Janev, L. P. Presnyakov, V.P. Shevelko, Physics of Highly Charged Ions, Springer Series in Electrophysics, 13, Springer, Berlin, 1985.

[5] Е. А. Соловьев, "Неадиабатические переходы в атомных столкновениях", УФН, 157:3 (1989), 437-476. 
[6] M. J. Jamieson, A. Dalgarno, M. Aymar, J. Tharamel, "A study of exchange interactions in alkali molecular ion dimers with application to charge transfer in cold Cs", J. Phys. B, 42:9 (2009), 095203, 10 pp.

[7] M. Hnatič, V. M. Khmara, V. Yu. Lazur, O. K. Reity, "Quasiclassical study of the quantum mechanical two-coulomb-centre problem", EPJ Web of Conf., 108 (2016), 02028.

[8] И. В. Комаров, Е. А. Соловьев, "Квазипересечения термов в задаче двух кулоновских центров с сильно отличающимися зарядами", ТМФ, 40:1 (1979), 130-136.

[9] T. P. Grozdanov, R. K. Janev, V. Yu. Lazur, "Asymptotic theory of the strongly asymmetric two-Coulomb-center problem", Phys. Rev. A, 32:6 (1985), 3425-3434.

[10] М. И. Карбованец, В. Ю. Лазур, М. И. Чибисов, "Нерезонансный обмен двумя электронами", ЖЭТФ, 86:1 (1984), 84-89.

[11] В. Ю. Лазур, Ю. Ю. Машика, Р. К. Янев, Т. П. Грозданов, "Квазипересечения ридберговских термов в задаче двух кулоновских центров с сильно отличающимися зарядами", ТМФ, 87:1 (1991), 97-109.

[12] П. П. Горват, В. Ю. Лазур, С. И. Мигалина, И. М. Шуба, Р. К. Янев, "Расщепление термов в квантово-механической задаче двух центров для уравнения Дирака", ТМФ, 109:2 (1996), 232-249.

[13] O. K. Reity, V. Yu. Lazur, A. V. Katernoha, "The quantum mechanical two-Coulomb-centre problem in the Dirac equation framework", J. Phys. B, 35:1 (2002), 1-17.

[14] Д. И. Бондарь, М. Гнатич, В. Ю. Лазур, “Двумерная задача двух кулоновских центров при малых межцентровых расстояниях", ТМФ, 148:2 (2006), 269-287.

[15] D. I. Bondar, M. Hnatich, V. Yu. Lazur, "The two Coulomb centres problem at small intercentre separations in the space of arbitrary dimension", J. Phys. A: Math. Theor., 40:8 (2007), 1791-1807.

[16] Д. И. Бондарь, М. Гнатич, В. Ю. Лазур, “Символьные вычисления для задачи двух кулоновских центров в пространстве произвольной размерности (англ)", Писвма в ЭЧАЯ, $\mathbf{5}: 3(145)(2008), 437-443$.

[17] Е. Е. Никитин, С. Я. Уманский, Неадиабатические переходы при медленных атомных столкновениях, Атомиздат, М., 1979.

[18] О.Б. Фирсов, "Резонансная перезарядка ионов при медленных столкновениях", ЖЭТФ, 21:3 (1951), 1001-1008.

[19] Т. М. Кереселидзе, М. И. Чибисов, “Асимптотическое поведение двухцентровой кулоновской волновой функции", Физика электронных и атомных столкновений, ЛФТИ, Л., 1989, 160-165.

[20] Б. М. Смирнов, Асимптотические методы в теории атомных столкновений, Атомиздат, М., 1973.

[21] Е. Е. Никитин, Б. М. Смирнов, Медленные атомные столкновения, Энергоатомиздат, M., 1990.

[22] M. I. Chibisov, R. K. Janev, "Asymptotic exchange interactions in ion-atom systems", Phys. Rep., 166:1 (1988), 1-87.

[23] M. B. Kadomtsev, S.I. Vinitsky, "Perturbation theory within the $\mathrm{O}(4,2)$ group for a hydrogen-like atom in the field of a distant charge", J. Phys. A: Math. Gen., 18:12 (1985), L689-L695.

[24] Е. А. Соловьев, "Приближенный интеграл движения для атома водорода в магнитном поле", Писъма в ЖЭТФ, 34:5 (1981), 278-281.

[25] T. M. Kereselidze, Z. S. Machavariani, I. L. Noselidze, "The one-Coulomb-centre problem in the spheroidal coordinate system: asymptotic solutions", J. Phys. B, 29:2 (1996), 257-273.

[26] T. M. Kereselidze, I. L. Noselidze, M. I. Chibisov, "Analytical expressions for two-Coulomb-centre wavefunctions at large internuclear distances", J. Phys. B, 36:5 (2003), 853-871. 
[27] Л. Д. Ландау, Е. М. Лифшиц, Курс теоретической физики, т. Квантовая механика (нерелятивистская теория), 3, Наука, М., 1989.

[28] Дж. Хединг, Введение в метод фазовых интегралов (метод ВКБ), Мир, М., 1965.

[29] Н. Фрёман, П. У. Фрёман, ВКБ-приближение, Мир, М., 1967.

[30] Л.И. Пономарев, "Об асимптотических представлениях сфероидальных функций", Ж. вычисл. матем. и матем. физ., 7:1 (1967), 196-198.

[31] M. V. Berry, K.E. Mount, "Semiclassical approximations in wave mechanics", Rep. Progr. Phys., 35:1 (1972), 315-397. 